Bull. Korean Math. Soc. 51 (2014), No. 5, pp. 1433-1451

http://dx.doi.org/10.4134/BKMS.2014.51.5.1433

\title{
MULTIPLE PERIODIC SOLUTIONS OF SECOND-ORDER ORDINARY DIFFERENTIAL EQUATIONS ACROSS RESONANCE
}

\author{
Hua Cai, Xiaojun Chang, and Xin Zhao
}

\begin{abstract}
In this paper we study the existence of multiple periodic solutions of second-order ordinary differential equations. New results of multiplicity of periodic solutions are obtained when the nonlinearity may cross multiple consecutive eigenvalues. The arguments are proceeded by a combination of variational and degree theoretic methods.
\end{abstract}

\section{Introduction and main results}

This paper is devoted to the existence of multiple periodic solutions for the second-order nonlinear ordinary differential equation

$$
\left\{\begin{array}{l}
-\ddot{x}=f(t, x), \\
x(0)=x(2 \pi), \dot{x}(0)=\dot{x}(2 \pi),
\end{array}\right.
$$

where $f \in C\left(\mathbb{R}^{2}, \mathbb{R}\right), f(t+T, x)=f(t, x)$ for all $(t, x) \in \mathbb{R}^{2}, T>0, f(t, 0)=0$. Clearly, the problem (1.1) has a trivial solution $x=0$. Our goal is to obtain multiple nontrivial periodic solutions of problem (1.1) when the nonlinearity $f$ grows linearly and the "ratio" $\frac{2 F(t, s)}{s^{2}}$ stays asymptotically at infinity between two consecutive eigenvalues of linear periodic boundary value problem

$$
\left\{\begin{array}{l}
-\ddot{x}=\lambda x \\
x(0)=x(2 \pi), \dot{x}(0)=\dot{x}(2 \pi),
\end{array}\right.
$$

where $F(t, s) \equiv \int_{0}^{s} f(t, \tau) d \tau$. The eigenvalues $\left\{k^{2}\right\}_{k \in \mathbb{N}}$ of the problem (1.2) are usually called resonant points of the problem (1.1).

Various works in the literature are devoted to resonant problems for ordinary or partial differential equations during the last several decades since the pioneer work Landesman and Lazer [21]. In these papers growth restrictions have been required on both the ratios $\frac{f(t, s)}{s}$ and $\frac{2 F(t, s)}{s^{2}}$ to control the interference of the nonlinearity with the eigenvalues of the associated linear problem (see

Received June 4, 2013.

2010 Mathematics Subject Classification. 34C25, 58E30.

Key words and phrases. periodic solutions, ordinary differential equations, resonance, variational method, Leray-Schauder degree. 
$[4,5,8,9,10,15,17,18,20,23,26])$. Among these works, Fabry and Fonda [15], Omari and Zanolin [26] established the solvability of (1.1) under the following so-called double-resonance conditions

$$
k^{2} \leq \liminf _{|s| \rightarrow \infty} \frac{f(t, s)}{s} \leq \limsup _{|s| \rightarrow \infty} \frac{f(t, s)}{s} \leq(k+1)^{2}
$$

for some $k \in \mathbb{N}$ and uniformly for a.e. $t \in[0,2 \pi]$ and some additional conditions imposed on $f$. Papageorgion and Staicu [27], Su and Zhao [28] studied the existence of multiple periodic solutions for problem (1.1) when double resonance occurs, where they assumed (1.3) and some generalization of the well-known Landesman-Lazer conditions (LL-conditions for short), see Landesman-Lazer [21], Fabry-Fonda [15] and Iannacci-Nkashama [20]. Under the additional assumption (1.6) or $f^{\prime}(t, 0)<0$ with $f$ being $C^{1}$, they both established the existence of at least two nontrivial solutions of (1.1). Recently, Barletta and Papageorgiou [2] used the variational methods together with Morse theory to obtain six nontrivial solutions of problem (1.1) when the nonlinearity is resonant both at infinity and at zero. In this paper, we shall show that if (1.3) with LL-conditions are replaced by the assumptions (1.4) and (1.5), problem (1.1) still admits at least two nontrivial solutions. Furthermore, if (1.4) and (1.5) are replaced by some stronger conditions (1.7) and (1.8), then we may obtain more nontrivial solutions. Note that in our results we don't require the ratio $\frac{f(t, s)}{s}$ stays asymptotically at infinity between two consecutive eigenvalues of (1.2), so the ratio $\frac{f(t, s)}{s}$ may cross resonant points $\left\{k^{2}\right\}$ asymptotically. Our method is a combination of variational method and degree theory.

Besides the above cited papers, for related works on resonant problems we also refer the interested reader to see $[6,7,12,13,14,16,23,29,30]$ etc and the references therein.

Let $H_{[0,2 \pi]}^{1}$ denote the Sobolev space

$$
\begin{gathered}
H_{[0,2 \pi]}^{1}=\{x:[0,2 \pi] \rightarrow \mathbb{R} \mid x \text { is absolutely continuous, } x(0)=x(2 \pi), \\
\left.\qquad \int_{0}^{2 \pi}|\dot{x}|^{2}+|x|^{2} d t<\infty\right\}
\end{gathered}
$$

equipped with the usual inner product and norm

$$
(x, y)=\int_{0}^{2 \pi}(\dot{x} \dot{y}+x y) d t,\|x\|=(x, x)^{\frac{1}{2}}, \forall x, y \in H_{[0,2 \pi]}^{1} .
$$

Define the functional $I: H_{[0,2 \pi]}^{1} \rightarrow \mathbb{R}$ by

$$
I(x)=\int_{0}^{2 \pi}\left[\frac{1}{2}|\dot{x}(t)|^{2}-F(t, x)\right] d t .
$$

Obviously, $I \in C^{1}\left(H_{[0,2 \pi]}^{1}, \mathbb{R}\right)$ (see [24]) and

$$
[\nabla I(x)](y)=\int_{0}^{2 \pi}[\dot{x} \dot{y}-f(t, x) y] d t, \forall x, y \in H_{[0,2 \pi]}^{1} .
$$


It is well known that $x$ is a weak solution of (1.1) if and only if $x$ is a critical point of the functional $I$.

Our main results are as follows.

Theorem 1.1. Assume that $f \in C([0,2 \pi] \times \mathbb{R}, \mathbb{R})$ and the following conditions hold:

(i) there exist $\eta_{1}, M, M_{1}>0$ such that

$$
\eta_{1} \leq \frac{f(t, s)}{s} \leq M \text { for }|s| \geq M_{1} \text {, a.e. } t \in[0,2 \pi]
$$

$$
k^{2} \leq l(t) \equiv \liminf _{|s| \rightarrow \infty} \frac{2 F(t, s)}{s^{2}} \leq \limsup _{|s| \rightarrow \infty} \frac{2 F(t, s)}{s^{2}} \equiv r(t) \leq(k+1)^{2}
$$

for a.e. $t \in[0,2 \pi]$ and some $k \in \mathbb{Z}^{+}$;

(iii) there exists $s_{1}>0$ such that

$$
F(t, s) \leq 0
$$

for a.e. $t \in[0,2 \pi]$ and all $s \in \mathbb{R}$ with $|s| \leq s_{1}$.

Then the problem (1.1) admits at least one nontrivial nonnegative solution and one nontrivial nonpositive solution.

Theorem 1.2. Assume that $f \in C([0,2 \pi] \times \mathbb{R}, \mathbb{R})$ with $f(t, x)=g(x)+e(t)$ and (1.6) holds. Suppose that the following conditions hold:

(i) there exist $\eta_{2}, C, C_{1}>0$ such that

$$
\eta_{2} \leq \frac{g(s)}{s} \leq C \text { for }|s| \geq C_{1}
$$

(ii) there exist $p, q>0$ such that

$$
k^{2}<p \leq l(t) \leq r(t) \leq q<(k+1)^{2}
$$

for a.e. $t \in[0,2 \pi]$ and some $k \in \mathbb{Z}^{+}$.

Then we have the following results:

(i) if $k$ is odd, then the problem (1.1) possesses at least two nontrivial solutions; if $k$ is even, then the problem (1.1) possesses at least three nontrivial solutions, one of which is nonnegative, and another one is nonpositive;

(ii) if all the critical points of the functional $I$ is nondegenerate, then the problem (1.1) admits at least four nontrivial solutions;

(iii) if $g \in C^{1}(\mathbb{R}, \mathbb{R})$ and there exists $\beta>0$ such that

$$
g^{\prime}(s) \leq \beta<(k+1)^{2}
$$

for all $s \in \mathbb{R}$, then the problem (1.1) admits at least four nontrivial solutions.

This paper is organized as follows. In $\S 2$, the proof of Theorem 1.1 is presented. In $\S 3$, we give the proof of Theorem 1.2 .

For convenience, we introduce some notations and definitions. $L^{p}(0,2 \pi)(1<$ $p<\infty)$ denotes the usual Sobolev space with inner product $\langle,\rangle_{p}$ and norm $\|\cdot\|_{p}$ 
respectively. $C^{m}[0,2 \pi]$ denotes the space of $m$-times continuous differential real functions with norm

$$
\|x\|_{C^{m}}=\max _{t \in[0,2 \pi]}|x(t)|+\max _{t \in[0,2 \pi]}|\dot{x}(t)|+\cdots+\max _{t \in[0,2 \pi]}\left|x^{(m)}(t)\right| .
$$

Note the eigenvalues $\left\{k^{2}\right\}_{k \in \mathbb{N}}$ are simple, and we denote $E\left(k^{2}\right)(k \in \mathbb{N})$ the eigenspace corresponding to $k^{2}$. Let

$$
V=E\left(0^{2}\right) \oplus E\left(1^{2}\right) \oplus \cdots \oplus E\left(k^{2}\right), W=\overline{E\left((k+1)^{2}\right) \oplus E\left((k+2)^{2}\right) \oplus \cdots} .
$$

Then

$$
H_{[0,2 \pi]}^{1}=V \oplus W
$$

and we can write $x \in H_{[0,2 \pi]}^{1}$ as $x=v+w$, where

$$
\begin{aligned}
& v(t)=a_{0}+\sum_{i=1}^{k}\left(a_{i} \cos (i t)+b_{i} \sin (i t)\right) \in V, \\
& w(t)=\sum_{i=k+1}^{\infty}\left(a_{i} \cos (i t)+b_{i} \sin (i t)\right) \in W .
\end{aligned}
$$

\section{Proof of Theorem 1.1}

Let $f^{+}:[0,2 \pi] \times \mathbb{R} \rightarrow \mathbb{R}$ be the function defined by

$$
f^{+}(t, s)= \begin{cases}f(t, s)+s, & s \geq 0 \\ 0, & s<0\end{cases}
$$

and $F^{+}(t, s)=\int_{0}^{s} f^{+}(t, \tau) d \tau$. Define the functional $I^{+}: H_{[0,2 \pi]}^{1} \rightarrow \mathbb{R}$ as follows

$$
I_{1}^{+}(x)=\int_{0}^{2 \pi}\left[\frac{1}{2}\left(|\dot{x}|^{2}+|x|^{2}\right)-F^{+}(t, x)\right] d t .
$$

By (1.4), $I_{1}^{+} \in C^{1}\left(H_{[0,2 \pi]}^{1}, \mathbb{R}\right)$. We assume that the set of all critical points of $I_{1}^{+}$is finite. In what follows, to obtain the critical points of $I_{1}^{+}$, we first show that the functional $I_{1}^{+}$has a mountain pass geometry. Precisely, since $H_{[0,2 \pi]}^{1}$ is embedded compactly into $C[0,2 \pi]$, we can find $c_{0}>0$ such that

$$
\|x\|_{\infty} \leq c_{0}\|x\| \text { for all } x \in H_{[0,2 \pi]}^{1} .
$$

Set $\delta_{0}=\frac{s_{1}}{c_{0}}$. Then, for all $x \in H_{[0,2 \pi]}^{1}$ with $\|x\| \leq \delta_{0}$, we get

$$
|x(t)| \leq c_{0}\|x\| \leq s_{1}, \text { a.e. } t \in[0,2 \pi]
$$

In view of (1.6), we have

$$
F^{+}(t, x) \leq 0 \text { for all }\|x\| \leq \delta_{0} \text {, a.e. } t \in[0,2 \pi] .
$$

Hence, for $x \in \bar{B}_{\delta_{0}}$ with $B_{\delta_{0}} \equiv\left\{x \in H_{[0,2 \pi]}^{1} \mid\|x\|<\delta_{0}\right\}$, it follows that

$$
I_{1}^{+}(x) \geq \int_{0}^{2 \pi} \frac{1}{2}|\dot{x}|^{2} d t \geq 0=I_{1}^{+}(0),
$$


which implies 0 is a local minimizer of $I_{1}^{+}$. Since we assume that $I_{1}^{+}$has finite critical points, we can find $\delta>0$ such that

$$
\inf _{\partial B_{\delta}} I_{1}^{+}>0=I_{1}^{+}(0) \text {. }
$$

Note that

$$
I_{1}^{+}(s)=-\int_{0}^{2 \pi} F^{+}(t, s) d t \text { for } s \in \mathbb{R}^{+} .
$$

By (1.5) it follows that

$$
I_{1}^{+}(s) \rightarrow-\infty \text { as } s \rightarrow \infty .
$$

Specially, there exists $x_{0} \in H_{[0,2 \pi]}^{1}$ with $\left\|x_{0}\right\|>\delta_{0}$ such that

$$
I_{1}^{+}\left(x_{0}\right)<0 .
$$

In what follows we come to prove that $I_{1}^{+}$satisfies the (PS) condition. Assume that $\left\{x_{n}\right\}_{n \in \mathbb{N}} \subset H_{[0,2 \pi]}^{1}$ is a sequence such that for some $M>0$,

$$
\left|I_{1}^{+}\left(x_{n}\right)\right| \leq M, \nabla I_{1}^{+}\left(x_{n}\right) \rightarrow 0 \text { as } n \rightarrow \infty .
$$

It suffices to prove that $\left\{x_{n}\right\}_{n \in \mathbb{N}}$ is bounded in $H_{[0,2 \pi]}^{1}$. Then a standard argument shows that $\left\{x_{n}\right\}_{n \in \mathbb{N}}$ has a convergent subsequence, which implies that $I_{1}^{+}$satisfies the (PS) condition. On the contrary, suppose that $\left\{x_{n}\right\}_{n \in \mathbb{N}}$ is unbounded in $H_{[0,2 \pi]}^{1}$, i.e.,

$$
\left\|x_{n}\right\| \rightarrow \infty \text { as } n \rightarrow \infty \text {. }
$$

Let $z_{n}=\frac{x_{n}}{\left\|x_{n}\right\|}$. Then $\left\|z_{n}\right\|=1$ and upon passing to a subsequence, there exists some $z_{0} \in H_{[0,2 \pi]}^{1}$ such that

$$
z_{n} \rightarrow z_{0} \text { in } H_{[0,2 \pi]}^{1}, z_{n} \rightarrow z_{0} \text { uniformly on }[0,2 \pi] .
$$

In view of $(2.3)$, for all $\phi \in H_{[0,2 \pi]}^{1}$, we have

$$
\int_{0}^{2 \pi}\left[\dot{z}_{n} \dot{\phi}+z_{n} \phi-\frac{f^{+}\left(t, x_{n}\right)}{\left\|x_{n}\right\|} \phi\right] d t \rightarrow 0 \text { as } n \rightarrow \infty .
$$

By (1.4), $\frac{f^{+}\left(t, x_{n}\right)}{\left\|x_{n}\right\|}$ remains bounded in $L^{2}[0,2 \pi]$. Thus, for a subsequence $\frac{f^{+}\left(t, x_{n}\right)}{\left\|x_{n}\right\|}$ converges weakly in $L^{2}[0,2 \pi]$ to some $\tilde{f}^{+} \in L^{2}[0,2 \pi]$ and by standard arguments based on (1.4), $\tilde{f}^{+}$can be written as

$$
\tilde{f}^{+}(t)=m(t) z_{0}(t)
$$

where $m \in L^{\infty}(0,2 \pi)$ satisfies

$$
|m(t)| \leq C \text { for a.e. } t \in[0,2 \pi] .
$$

Consequently, by (2.5)-(2.7), $z_{0}$ is a solution of

$$
\left\{\begin{array}{l}
-\ddot{z}_{0}+z_{0}=m(t) z_{0} \text { in }[0,2 \pi], \\
z_{0}(0)=z_{0}(2 \pi), \quad \dot{z}_{0}(0)=\dot{z}_{0}(2 \pi) .
\end{array}\right.
$$


Define

$$
\begin{aligned}
& \Omega_{+}=\left\{t \in[0,2 \pi] \mid z_{0}(t)>0\right\}, \Omega_{-}=\left\{t \in[0,2 \pi] \mid z_{0}(t)<0\right\}, \\
& \Omega_{0}=\left\{t \in[0,2 \pi] \mid z_{0}(t)=0\right\} .
\end{aligned}
$$

Then we consider the three cases respectively.

(i) On $\Omega_{+}$. For $t \in \Omega_{+}$, we have

$$
x_{n}(t)=z_{n}(t)\left\|x_{n}\right\| \rightarrow+\infty \text { as } n \rightarrow+\infty,
$$

which implies that there exists $n_{1} \in \mathbb{Z}^{+}$such that $x_{n}(t)>M_{1}$ for all $t \in \Omega_{+}$if $n \geq n_{1}$. Hence by (1.4),

$$
\eta_{1}+1 \leq \frac{f^{+}\left(t, x_{n}(t)\right)}{x_{n}(t)} \leq M+1 \text { for } n \geq n_{1} .
$$

Then by standard arguments it follows that

$$
\eta_{1}+1 \leq m(t) \leq M+1 \text { for } t \in \Omega_{+} .
$$

(ii) On $\Omega_{-}$. For $t \in \Omega_{-}$, we have

$$
x_{n}(t)=z_{n}(t)\left\|x_{n}\right\| \rightarrow-\infty \text { as } n \rightarrow+\infty,
$$

which implies that there exists $n_{2} \in \mathbb{Z}^{+}$such that $x_{n}(t)<-M_{1}$ for all $t \in \Omega_{-}$ if $n \geq n_{2}$. Then we get

$$
f^{+}\left(t, x_{n}(t)\right) \equiv 0 \text { for } n \geq n_{1} .
$$

Hence

$$
m(t) \equiv 0 \text { for } t \in \Omega_{-} .
$$

(iii) On $\Omega_{0}$. Firstly by virtue of $(1.5)$ we can see that there exist $M_{2}>0$, $b_{1} \in L^{\infty}(0,2 \pi)$ such that

$$
\left|f\left(t, x_{n}(t)\right)\right| \leq M_{2}\left|x_{n}(t)\right|+b_{1}(t) .
$$

For any given $t \in \Omega_{0}$, if $\left|x_{n}(t)\right| \leq M_{1}$, then by (2.9) we have

$$
\left|f\left(t, x_{n}(t)\right)\right| \leq M_{2} M_{1}+b_{1}(t) \text {. }
$$

Since $\left\|x_{n}\right\| \rightarrow \infty$, we obtain that

$$
\frac{f^{+}\left(t, x_{n}\right)}{\left\|x_{n}\right\|} \rightarrow 0
$$

if $\left|x_{n}(t)\right| \geq M_{1}$, then

Hence we have

$$
0 \leq \frac{f^{+}\left(t, x_{n}\right)}{x_{n}} \leq M+1
$$

$$
0 \leq m(t) \leq M+1 \text { for } t \in \Omega_{0} .
$$

Now, acting on (2.8) with $z_{0}^{-}$we can get $z_{0} \geq 0$. Thus, meas $\left(\Omega_{-}\right)=0$. In addition, by the former of (2.3), we obtain

$$
\frac{\left|\nabla I_{1}^{+}\left(x_{n}\right) \cdot x_{n}\right|}{\left\|x_{n}\right\|^{2}}=\left|1-\int_{0}^{2 \pi} \frac{f^{+}\left(t, x_{n}\right)}{\left\|x_{n}\right\|} z_{n} d t\right| \rightarrow 0,
$$


so that

$$
\int_{0}^{2 \pi} \frac{f^{+}\left(t, x_{n}\right)}{x_{n}} z_{n}^{2} d t \rightarrow 1
$$

Then, by (2.5) and (2.7), we have

$$
\int_{0}^{2 \pi} m(t) z_{0}^{2}(t) d t=1
$$

Hence $z_{0} \not \equiv 0$. By modifying $m(t)$ on $\Omega_{0}$ if necessary, we may assume that $\eta_{1}+1 \leq m(t) \leq M+1$ on $\Omega_{0}$. Then we have

$$
\eta_{1}+1 \leq m(t) \leq M+1 \text { a.e. } t \in[0,2 \pi] \text {. }
$$

Integrating $(2.8)$ on $[0,2 \pi]$ we have $\int_{0}^{2 \pi}(m(t)-1) z_{0}(t) d t=0$, which implies that

$$
(m(t)-1) z_{0}(t)=0 \text { a.e. in }[0,2 \pi] .
$$

Acting on (2.8) with $z_{0}$ we get

$$
0 \leq \int_{0}^{2 \pi}\left|\dot{z}_{0}(t)\right|^{2} d t=\int_{0}^{2 \pi}(m(t)-1) z_{0}^{2}(t) d t=0 .
$$

Together with (2.11) and (2.12) we can see $z_{0} \equiv 0$ on $[0,2 \pi]$. A contradiction. Thus $\left\{x_{n}\right\}_{n \in \mathbb{N}}$ is bounded in $H_{[0,2 \pi]}^{1}$ and standard arguments imply that $I_{1}^{+}$ satisfies the (PS) condition. Therefore, by the mountain pass theorem, $I_{1}^{+}$ has a critical point $x^{+}$with $I_{1}^{+}\left(x^{+}\right) \geq\left. I_{1}^{+}(x)\right|_{\partial B_{\delta}}>0$. By the assumption of $f^{+}$we can see that $x^{+} \geq 0$ with $x^{+} \not \equiv 0$ and $x^{+}$is a nontrivial nonnegative critical point of $I$, which implies that (1.1) admits a nontrivial nonnegative weak solution $x^{+}$. Similar arguments show that (1.1) also admits a nontrivial nonpositive weak solution $x^{-}$. The proof is complete.

\section{Proof of Theorem 1.2}

Lemma 3.1. If 0 is an isolated critical point, then there exists $\rho_{0}$ small such that

$$
\operatorname{deg}\left(\nabla I, B_{\rho}, 0\right)=1 \text { for all } 0<\rho \leq \rho_{0} .
$$

Proof. Similar arguments as in the proof of Theorem 1.1 imply that 0 is a strict local minimizer of $I$. Then the conclusion is obtained by Corollary 2 of Amann [1].

Lemma 3.2. Under the assumption of Theorem 1.2, the problem (1.1) admits a nontrivial nonnegative solution $x^{+}$and a nontrivial nonpositive solution $x^{-}$. Furthermore, there exist $r_{1}, d_{1}>0$ small such that

$$
\operatorname{deg}\left(\nabla I, B_{r}\left(x^{+}\right), 0\right)=-1 \text { for all } 0<r \leq r_{1}
$$

and

$$
\operatorname{deg}\left(\nabla I, B_{d}\left(x^{-}\right), 0\right)=-1 \text { for all } 0<d \leq d_{1},
$$

where $B_{r}(x)=\left\{y \in H_{[0,2 \pi]}^{1} \mid\|y-x\|<r\right\}$. 
Proof. Note that (1.7) and (1.8) imply that (1.4) and (1.5). Then by Theorem $1.1,(1.1)$ possesses a nontrivial nonnegative solution $x^{+}$and a nontrivial nonpositive solution $x^{-}$. The arguments in the proof of Theorem 1.1 show that $x^{+}$ and $x^{-}$are both of mountain pass type. Then, by Theorems 1 and 2 of [19], they both have local degree -1 and (3.1), (3.2) hold.

Lemma 3.3. Assume that $f \in C([0,2 \pi] \times \mathbb{R}, \mathbb{R})$ and (1.7), (1.8) hold. If $x$ is a solution of (1.1), then there exists a constant $C_{2}>0$ such that

$$
\|x\|_{C^{1}} \leq C_{2} \text {. }
$$

Proof. We assume by the contradiction that there exists a sequence of $2 \pi$ periodic solutions $\left\{x_{n}(t)\right\}_{1}^{\infty}$ of (1.1) corresponding to sequence $\left\{\lambda_{n}\right\}_{1}^{\infty} \subset[0,1]$ such that

$$
\left\|x_{n}\right\|_{C^{1}} \rightarrow \infty \text { as } n \rightarrow \infty \text {. }
$$

Define $z_{n}(t)=\frac{x_{n}(t)}{\left\|x_{n}\right\|_{C^{1}}}$. Then $\left\|z_{n}\right\|_{C^{1}}=1$ and $z_{n}$ satisfies

$$
-\ddot{z}_{n}(t)=\frac{f\left(t, x_{n}\right)}{\left\|x_{n}\right\|_{C^{1}}} .
$$

By (1.7), there must be some $M>0$ such that

$$
\left|\ddot{z}_{n}(t)\right| \leq M, \forall t \in[0,2 \pi],
$$

which implies that $\left\{z_{n}(t)\right\}$ and $\left\{\dot{z}_{n}(t)\right\}$ are uniformly bounded and equicontinuous on $[0,2 \pi]$. Then by the Arzela-Ascoli theorem, taking subsequences if possible, it follows that

$$
z_{n}(t) \rightarrow \bar{z}(t), \dot{z}_{n}(t) \rightarrow \dot{\bar{z}}(t) \text { as } n \rightarrow \infty
$$

hold for some $\bar{z} \in C^{1}[0,2 \pi]$ with $\|\bar{z}(t)\|_{C^{1}}=1$ on $[0,2 \pi]$. We prove now the following fact.

Claim 1. There exists $d>0$ such that, for each $n$, there exists $t_{n} \in[0,2 \pi]$ such that

$$
\left|x_{n}\left(t_{n}\right)\right| \leq d
$$

Proof. Firstly, in view of (1.7), we can take $d>0$ large enough such that

$$
f(t,-d)<0<f(t, d), \forall t \in[0,2 \pi] .
$$

Secondly, we shall show that there exists $d>0$ satisfying (3.8) such that, for any $n$, the following conclusions hold.

$$
\min _{t \in[0,2 \pi]} x_{n}(t) \neq d, \max _{t \in[0,2 \pi]} x_{n}(t) \neq-d .
$$

We assume by contradiction that there is some $j \in \mathbb{Z}^{+}$and $t_{0} \in[0,2 \pi]$ such that $\min _{t \in[0,2 \pi]} x_{j}(t)=x_{j}\left(t_{0}\right)=d$. By (3.8), it follows that there exist $\epsilon_{j}, \delta_{j}>0$ such that

$$
-\ddot{x}_{j}(t)=f\left(t, x_{j}(t)\right) \geq \epsilon_{j}
$$


hold for $t \in\left[t_{0}-\delta_{j}, t_{0}+\delta_{j}\right]$. Thus, taking $t_{1} \in\left[t_{0}-\delta_{j}, t_{0}\right)$, by $\dot{x}_{j}\left(t_{0}\right)=0$, we have

$$
x_{j}\left(t_{1}\right)-x_{j}\left(t_{0}\right)=\int_{t_{1}}^{t_{0}} \ddot{x}_{j}(s)\left(s-t_{1}\right) d s<0,
$$

which is impossible. Thus the claim is right.

By the boundedness of the sequence $\left\{t_{n}\right\}$, passing to a subsequence if possible, there exists $\hat{t} \in[0,2 \pi]$ such that

$$
t_{n} \rightarrow \hat{t} \text { as } n \rightarrow \infty \text {. }
$$

Multiplying both sides of (3.5) by $\dot{z}_{n}(t)$ and integrating from $t_{n}$ to $t$, we have

$$
\begin{aligned}
{\left[\dot{z}_{n}\left(t_{n}\right)\right]^{2}-\left[\dot{z}_{n}(t)\right]^{2}=} & \frac{2 G\left(x_{n}(t)\right)}{x_{n}^{2}(t)}\left(z_{n}(t)\right)^{2}-\frac{2 G\left(x_{n}\left(t_{n}\right)\right)}{x_{n}}\left(z_{n}\left(t_{n}\right)\right)^{2} \\
& +\int_{t_{n}}^{t} \frac{e(s)}{\left\|x_{n}\right\|_{C^{1}}} \dot{z}_{n}(s) d s
\end{aligned}
$$

where $G(s)=\int_{0}^{s} g(\tau) d \tau$. By (3.7) it follows that

$$
\lim _{n \rightarrow \infty} z_{n}\left(t_{n}\right)=\lim _{m \rightarrow \infty} \frac{x_{n}\left(t_{n}\right)}{\left\|x_{n}\right\|_{C^{1}}}=0 .
$$

Taking a superior limit in (3.11), together with (3.6), (3.10) and (3.12), we can obtain that

$$
[\dot{\bar{z}}(\hat{t})]^{2}-[\dot{\bar{z}}(t)]^{2}=\limsup _{n \rightarrow \infty} \frac{2 G\left(x_{n}(t)\right)}{x_{n}^{2}(t)} \cdot \bar{z}^{2}(t) .
$$

Now by (1.8) it follows that

$$
[\dot{\bar{z}}(t)]^{2}-[\dot{\bar{z}}(\hat{t})]^{2}+q[\bar{z}(t)]^{2} \geq 0 .
$$

Similarly, we can obtain that

$$
[\dot{\bar{z}}(t)]^{2}-[\dot{\bar{z}}(\hat{t})]^{2}+p[\bar{z}(t)]^{2} \leq 0 .
$$

Hence, by (3.13)-(3.14), for $t \in[0,2 \pi]$, we have

$$
p[\bar{z}(t)]^{2} \leq[\dot{\bar{z}}(\hat{t})]^{2}-[\dot{\bar{z}}(t)]^{2} \leq q[\bar{z}(t)]^{2} .
$$

Claim 2. $\dot{\bar{z}}(t)$ has no zero accumulation points on $[0,2 \pi]$.

Proof. In fact, if not, we assume that there exists a sequence $\left\{\xi_{i}\right\} \subset[0,2 \pi]$ such that

$$
\dot{\bar{z}}\left(\xi_{i}\right)=0, \lim _{i \rightarrow \infty} \xi_{i}=\xi_{0} \in[0,2 \pi] .
$$

Clearly, $\dot{\bar{z}}\left(\xi_{0}\right)=0$. Taking $t=\xi_{i}$ in (3.15) and letting $i \rightarrow \infty$, it is not difficult to see that $\bar{z}\left(\xi_{0}\right) \neq 0$. Without loss of generality, we assume that $\bar{z}\left(\xi_{0}\right)>0$. By the continuity of $\bar{z}(t)$, there exist $\epsilon, \delta>0$ such that

$$
\bar{z}(t) \geq \epsilon, \forall t \in\left[\xi_{0}-\delta, \xi_{0}+\delta\right] .
$$

Then, there exists $n_{0} \in \mathbb{Z}^{+}$such that, for $n \geq n_{0}$,

$$
x_{n}(t) \geq C_{1}, \forall t \in\left[\xi_{0}-\delta, \xi_{0}+\delta\right] .
$$


In view of (1.7), (1.8), we can see that

$$
\frac{g\left(x_{n}(t)\right)}{x_{n}(t)} \geq \eta_{2}, \forall t \in\left[\xi_{0}-\delta, \xi_{0}+\delta\right] .
$$

Taking $\xi_{*}, \xi^{*} \in\left\{\xi_{i}\right\} \cap\left[\xi_{0}-\delta, \xi_{0}+\delta\right]$ and integrating from $\xi_{*}$ to $\xi^{*}$, we obtain that

$$
\dot{\bar{z}}_{n}\left(\xi_{*}\right)-\dot{\bar{z}}_{n}\left(\xi^{*}\right)=\int_{\xi_{*}}^{\xi^{*}} \frac{g\left(x_{n}(t)\right)+e(t)}{\left\|x_{n}\right\|_{C^{1}}} d t .
$$

Taking $n \rightarrow \infty$, by (3.16)-(3.17) we get

$$
0 \geq \eta_{2} \epsilon\left(\xi^{*}-\xi_{*}\right)>0 .
$$

A contradiction.

Now we come to show that (3.15) has only a trivial $2 \pi$ periodic solution. We assume by contradiction that (3.15) has a nontrivial $2 \pi$ periodic solution $z_{0}(t)$. In $(3.15)$, if $\dot{z}_{0}(\hat{t})=0$, then by (1.8) it follows that

$$
k^{2}\left[z_{0}(t)\right]^{2}<\left[\dot{z}_{0}(t)\right]^{2}<(k+1)^{2}\left[z_{0}(t)\right]^{2} .
$$

It is easily seen that $z_{0}(t) \equiv 0$ on $[0,2 \pi]$, which is contrary to that $\left\|z_{0}\right\|_{C^{1}}=1$. If $\dot{z}_{0}(\hat{t}) \neq 0$, without loss of generality, we suppose that $\hat{t}=0$ and $\dot{z}_{0}(0)>0$. Assume that $z_{1}(t)$ and $z_{2}(t)$ are solutions of the following equations, respectively

$$
[\dot{z}(t)]^{2}-[\dot{z}(0)]^{2}=-p[z(t)]^{2},[\dot{z}(t)]^{2}-[\dot{z}(0)]^{2}=-q[z(t)]^{2}
$$

with

$$
z_{0}(0)=z_{1}(0)=z_{2}(0), \dot{z}_{1}(0) \leq \dot{z}_{0}(0) \leq \dot{z}_{2}(0)
$$

Then we have

$$
z_{1}(t) \leq z_{0}(t) \leq z_{2}(t), t \in\left[0, t_{1}\right]
$$

where $t_{1}$ is the first zero point of $z_{0}(t)$ in $(0,2 \pi]$. Similarly, if $z_{1}(t)$ and $z_{2}(t)$ are respectively solutions of (3.18) with

$$
z_{0}\left(t_{1}\right)=z_{1}\left(t_{1}\right)=z_{2}\left(t_{1}\right), \dot{z}_{1}\left(t_{1}\right) \leq \dot{z}_{0}\left(t_{1}\right) \leq \dot{z}_{2}\left(t_{1}\right),
$$

then we have

$$
z_{1}(t) \leq z_{0}(t) \leq z_{2}(t), t \in\left[t_{1}, t_{2}\right]
$$

where $t_{2}$ is the first zero point of $z_{0}$ in $(0,2 \pi]$. Since $\dot{z}_{0}(t)$ has no zero accumulation point in $[0,2 \pi]$, it follows that $(3.18)$ are equivalent to the following equations

$$
-\ddot{z}(t)=p z(t),-\ddot{z}(t)=q z(t)
$$

respectively. Then there are positive constants $\rho_{1}, \rho_{2}, \rho_{3}, \rho_{4}$ such that

$$
\begin{array}{r}
\rho_{1} \sin (\sqrt{q} t) \leq z_{0}(t) \leq \rho_{2} \sin (\sqrt{p} t), \quad 0<t \leq t_{1}, \\
\rho_{3} \sin \left(\sqrt{q}\left(t-t_{1}\right)\right) \leq z_{0}(t) \leq \rho_{4} \sin \left(\sqrt{p}\left(t-t_{1}\right)\right), \quad t_{1} \leq t \leq t_{2},
\end{array}
$$


which implies that

$$
\frac{\pi}{\sqrt{q}} \leq t_{1} \leq \frac{\pi}{\sqrt{p}}, \frac{2 \pi}{\sqrt{q}} \leq t_{2} \leq \frac{2 \pi}{\sqrt{p}} .
$$

Hence, by virtue of the periodicity of $z_{0}(t)$, there exists some positive integer $m$ such that

$$
\frac{2 m \pi}{k+1}<\frac{2 m \pi}{\sqrt{q}} \leq t_{m}=2 \pi \leq \frac{2 m \pi}{\sqrt{p}}<\frac{2 m \pi}{k},
$$

which implies that $k<m<k+1$, a contradiction. Therefore, (3.4) is impossible and (3.3) holds. This completes the proof.

Denote $\alpha=\frac{k^{2}+(k+1)^{2}}{2}$. Then the following problem

$$
\left\{\begin{array}{l}
-\ddot{x}=\alpha x \text { in }[0,2 \pi] \\
x(0)=x(2 \pi), \dot{x}(0)=\dot{x}(2 \pi)
\end{array}\right.
$$

has the only trivial solution 0 . Define

$$
\Phi(x)=\frac{1}{2} \int_{0}^{2 \pi}\left[|\dot{x}|^{2}-\alpha x^{2}(t)\right] d t, \forall x \in H_{[0,2 \pi]}^{1}
$$

and

$$
N[x](y)=\int_{0}^{2 \pi}(\alpha+1) x y d x, \forall x, y \in H_{[0,2 \pi]}^{1} .
$$

Then $\Phi \in C^{2}\left(H_{[0,2 \pi]}^{1}\right)$ and $N$ is a compact linear operator from $H_{[0,2 \pi]}^{1}$ to $H_{[0,2 \pi]}^{1}$. Note that

$$
[\nabla \Phi(x)](y)=\int_{0}^{2 \pi}[\dot{x} \dot{y}+x y] d t-\int_{0}^{2 \pi}(\alpha+1) x y d x, \forall x, y \in H_{[0,2 \pi]}^{1} .
$$

Then we have

$$
\nabla \Phi=i d-N
$$

Denote $B_{R}=\left\{x \in H_{[0,2 \pi]}^{1} \mid\|x\|<R, R \in \mathbb{R}^{+}\right\}$. It is easily seen that 0 is the only critical point of $\Phi$. Then $\nabla \Phi(x) \neq 0$ for all $x \in \partial B_{R}$ with $R>0$. So $\nabla \Phi$ is well defined on $B_{R}$. Denote deg and $i n d e x_{L S}$ as Leray-Schauder degree and index respectively. $i d$ denotes the identical mapping. We have the following result.

Lemma 3.4. There exists $R_{0}>0$ such that

$$
\operatorname{deg}\left(\nabla \Phi, B_{R}, 0\right)=(-1)^{k}, \forall R \geq R_{0} .
$$

Proof. From Theorem 2.8.1 in [25], it follows that

$$
\begin{aligned}
\operatorname{deg}\left(\nabla \Phi, B_{R}, 0\right) & =\operatorname{deg}\left(i d-N, B_{0}, 0\right) \\
& =\operatorname{index}_{L S}(i d-N, 0) \\
& =(-1)^{\beta}
\end{aligned}
$$


where

$$
\beta=\sum_{\lambda_{j}>1, \lambda_{j} \in \sigma(N)} \beta_{j}, \beta_{j}=\operatorname{dim} \cup_{i=1}^{\infty} \operatorname{ker}\left(\lambda_{j} \cdot i d-N\right)^{i} .
$$

Hence we just need to compute index ${ }_{L S}(i d-N, 0)$. If $N x=\lambda x$ for some $\lambda \in \mathbb{R}$ and $x \neq 0$, then we have

$$
(N x, y)=\int_{0}^{2 \pi}(\alpha+1) x y d t=\int_{0}^{2 \pi} \lambda[\dot{x} \dot{y}+x y] d t, \forall x, y \in H_{[0,2 \pi]}^{1},
$$

which together with (1.1) implies that $\lambda=\frac{\alpha+1}{n^{2}+1}$ for some $n \in \mathbb{Z}^{+}$. By $k^{2}<$ $\alpha<(k+1)^{2}$, we can obtain that $\lambda>1$ holds for all $n \leq k$, which implies that $\operatorname{deg}\left(\nabla \Phi, B_{R}, 0\right)=(-1)^{k}$ for all $R \geq R_{0}$. This completes the proof.

Apply above lemma, we can obtain the following result.

Lemma 3.5. Under the assumption of Theorem 1.2, there exists $R_{1}>0$ such that

$$
\operatorname{deg}\left(\nabla I, B_{R}, 0\right)=(-1)^{k}, \forall R \geq R_{1} .
$$

Proof. Consider the following auxiliary problem

$$
\left\{\begin{array}{l}
-\ddot{x}=\lambda f(t, x)+(1-\lambda) \alpha x \equiv f_{\lambda}(t, x), \text { a.e. } t \in[0,2 \pi], \lambda \in[0,1], \\
x(0)=x(2 \pi), \dot{x}(0)=\dot{x}(2 \pi) .
\end{array}\right.
$$

Define

$$
I_{\lambda}(x)=\int_{0}^{2 \pi}\left[\frac{1}{2}|\dot{x}(t)|^{2} d t-F_{\lambda}(t, x)\right] d t,
$$

where $F_{\lambda}(t, x)=\int_{0}^{x} f_{\lambda}(t, s) d s$. By (1.4)-(1.5) and the definition of $\alpha$, we can see that the conditions of Lemma 3.3 hold, so there exists $R_{1}>0$ large enough such that, for all $R \geq R_{1},\left.\nabla I_{\lambda}\right|_{\partial B_{R}}(x) \neq 0$ uniformly for $\lambda \in[0,1]$. Then by the homotopy invariance of the Leray-Schauder degree and Lemma 3.4, we obtain that

$$
\begin{aligned}
\operatorname{deg}\left(\nabla I, B_{R}, 0\right) & =\operatorname{deg}\left(\nabla I_{1}, B_{R}, 0\right) \\
& =\operatorname{deg}\left(\nabla I_{0}, B_{R}, 0\right) \\
& =\operatorname{deg}\left(\nabla \Phi, B_{R}, 0\right) \\
& =(-1)^{k}, \forall R \geq R_{1} .
\end{aligned}
$$

Proof of Theorem 1.2(i). Suppose that the set of all critical points of the functional $I$ is finite. If $k$ is odd, then by Lemma 3.2 we have obtained two nontrivial solutions. So we just prove the case of $k$ is even. Choose $\rho \in\left(0, \rho_{0}\right), r \in\left(0, r_{1}\right)$, $d \in\left(0, d_{1}\right)$ and $R \geq R_{0}$ such that

$$
B_{\rho} \cap B_{r}\left(x^{+}\right)=\emptyset, B_{\rho} \cap B_{d}\left(x^{-}\right)=\emptyset, B_{r}\left(x^{+}\right) \cap B_{d}\left(x^{-}\right)=\emptyset
$$

and

$$
\bar{B}_{\rho}, \bar{B}_{r}\left(x^{+}\right), \bar{B}_{d}\left(x^{-}\right) \subset B_{R}
$$


Then from the additivity and excision properties of the Leray-Schauder degree, we have

$$
\begin{aligned}
\operatorname{deg}\left(\nabla I, B_{R}, 0\right)= & \operatorname{deg}\left(\nabla I, B_{\rho}, 0\right)+\operatorname{deg}\left(\nabla I, B_{r}\left(x^{+}\right), 0\right)+\operatorname{deg}\left(\nabla I, B_{d}\left(x^{-}\right), 0\right) \\
& +\operatorname{deg}\left(\nabla I, B_{R} \backslash\left(\overline{B_{\rho} \cup B_{r}\left(x^{+}\right) \cup B_{d}\left(x^{-}\right)}\right), 0\right) .
\end{aligned}
$$

In view of Lemmas 3.1, 3.2 and 3.5, we obtain

$$
(-1)^{k}=1+(-1)+(-1)+\operatorname{deg}\left(\nabla I, B_{R} \backslash\left(\overline{B_{\rho} \cup B_{r}\left(x^{+}\right) \cup B_{d}\left(x^{-}\right)}\right), 0\right),
$$

which together with the excision property of the Leray-Schauder degree implies that

$$
\operatorname{deg}\left(\nabla I, B_{R} \backslash\left(\overline{B_{\rho} \cup B_{r}\left(x^{+}\right) \cup B_{d}\left(x^{-}\right)}\right), 0\right)=1+(-1)^{k} .
$$

Then if $k$ is even, it follows that

$$
\operatorname{deg}\left(\nabla I, B_{R} \backslash\left(\overline{B_{\rho} \cup B_{r}\left(x^{+}\right) \cup B_{d}\left(x^{-}\right)}\right), 0\right)=2 .
$$

Hence, by the existence property of the Leray-Schauder degree we can see that there exists $x^{*} \in B_{R} \backslash\left(\overline{B_{\rho} \cup B_{r}\left(x^{+}\right) \cup B_{d}\left(x^{-}\right)}\right)$such that $\nabla I\left(x^{*}\right)=0$, which together with $x^{+}, x^{-}$gives the existence of at least three nontrivial solutions of problem (1.1). This completes the proof.

Proof of Theorem 1.2(ii). Firstly we shall show that the functional $I$ satisfies the hypotheses of the saddle point theorem. In fact, since $f$ is continuous and satisfies (1.7) it follows that $I \in C^{1}\left(H_{[0,2 \pi]}^{1}, \mathbb{R}\right)$. By (1.8), there exist $\epsilon_{1}, \epsilon_{2}>0$ satisfying

$$
k^{2}<p-\epsilon_{1} \leq l(t) \leq r(t) \leq q+\epsilon_{2}<(k+1)^{2} \text { a.e. } t \in[0,2 \pi]
$$

and $a_{\epsilon_{1}}, b_{\epsilon_{2}} \in L^{1}(0,2 \pi)$ such that

$$
\left(p-\epsilon_{1}\right) s^{2}-a_{\epsilon_{1}}(t) \leq 2 F(t, s) \leq\left(q+\epsilon_{2}\right) s^{2}+b_{\epsilon_{2}}(t) \text { a.e. } t \in[0,2 \pi], \forall s \in \mathbb{R} .
$$

Then, we have

$$
\begin{gathered}
\int_{0}^{2 \pi} \frac{1}{2}\left[|\dot{x}|^{2}-\left(q+\epsilon_{2}\right) x^{2}+2 b_{\epsilon_{2}}(t)\right] d t \\
\leq I(x) \leq \int_{0}^{2 \pi} \frac{1}{2}\left[|\dot{x}|^{2}-\left(p-\epsilon_{1}\right) x^{2}+2 a_{\epsilon_{1}}(t)\right] d t .
\end{gathered}
$$

We claim that

$$
I(w) \rightarrow \infty \text { as }\|w\| \rightarrow \infty \text { for } w \in W .
$$

Since for $w \in W$,

we get

$$
\int_{0}^{2 \pi} \dot{w}^{2}(t) d t \geq(k+1)^{2} \int_{0}^{2 \pi} w^{2}(t) d t
$$

$$
\|w\|^{2}=\int_{0}^{2 \pi}\left[|\dot{w}|^{2}+w^{2}\right] d t \leq\left(1+\frac{1}{(k+1)^{2}}\right) \int_{0}^{2 \pi} \dot{w}^{2}(t) d t
$$


Then, we can obtain by (3.22) and (3.24) that

$$
\begin{aligned}
I(w) & \geq \frac{1}{2}\left[1-\frac{q+\epsilon_{2}}{(k+1)^{2}}\right] \int_{0}^{2 \pi}|\dot{w}|^{2} d t-2\left\|b_{\epsilon_{2}}\right\|_{L^{2}(0,2 \pi)} \\
& \geq \delta_{1}\|w\|^{2}-2\left\|b_{\epsilon_{2}}\right\|_{L^{2}(0,2 \pi)}
\end{aligned}
$$

where $\delta_{1}=\frac{1}{2}\left[1-\frac{q+\epsilon_{2}}{(k+1)^{2}}\right] \frac{1}{1+\frac{1}{(k+1)^{2}}}$. By $(3.21), \delta_{1}>0$. Thus $I$ satisfies (3.23).

Now we claim that

$$
I(v) \rightarrow-\infty \text { as }\|v\| \rightarrow \infty \text { for } v \in V .
$$

Write

$$
V=V_{0} \oplus V_{1}
$$

where $V_{0}=E\left(0^{2}\right), V_{1}=E\left(1^{2}\right) \oplus E\left(2^{2}\right) \oplus \cdots \oplus E\left(k^{2}\right)$. Then we can write for each $v \in V$ as $v=v_{0}+v_{1}$, where $v_{0} \in V_{0}, v_{1} \in V_{1}$. Note for $v_{1} \in V_{1}$,

We have

$$
\int_{0}^{2 \pi} v_{1}^{2}(t) d t \leq \int_{0}^{2 \pi} \dot{v}_{1}^{2}(t) d t \leq k^{2} \int_{0}^{2 \pi} v_{1}^{2}(t) d t
$$

$$
\left(1+\frac{1}{k^{2}}\right) \int_{0}^{2 \pi} \dot{v}_{1}^{2}(t) d t \leq\left\|v_{1}\right\|^{2}=\int_{0}^{2 \pi}\left[\dot{v}_{1}^{2}(t)+v_{1}^{2}(t)\right] d t \leq 2 \int_{0}^{2 \pi} \dot{v}_{1}^{2}(t) d t
$$

Then we obtain by (3.21), (3.22) and (3.26) that, for $v=v_{0}+v_{1} \in V$,

$$
\begin{aligned}
I(v) & =\int_{0}^{2 \pi} \frac{1}{2}\left[|\dot{v}|^{2}-\frac{1}{2}\left(p-\epsilon_{1}\right) v^{2}+a_{\epsilon_{1}}(t)\right] d t . \\
& =\frac{1}{2} \int_{0}^{2 \pi}\left|\dot{v}_{1}\right|^{2} d t-\frac{1}{2}\left(p-\epsilon_{1}\right)\left[\int_{0}^{2 \pi} v_{1}^{2} d t+\int_{0}^{2 \pi} v_{0}^{2} d t\right]+\left\|a_{\epsilon_{1}}\right\|_{L^{2}(0,2 \pi)} \\
& \leq \frac{1}{2}\left(1-\frac{p-\epsilon_{1}}{k^{2}}\right) \int_{0}^{2 \pi}\left|\dot{v}_{1}\right|^{2} d t-\frac{1}{2}\left(p-\epsilon_{1}\right) \int_{0}^{2 \pi} v_{0}^{2} d t+\left\|a_{\epsilon_{1}}\right\|_{L^{2}(0,2 \pi)} \\
& \leq \frac{1}{4}\left(1-\frac{p-\epsilon_{1}}{k^{2}}\right)\left\|v_{1}\right\|^{2}-\frac{1}{2}\left(p-\epsilon_{1}\right)\left\|v_{0}\right\|^{2}+\left\|a_{\epsilon_{1}}\right\|_{L^{2}(0,2 \pi)} \\
& \leq-\delta_{2}\|v\|^{2}+\left\|a_{\epsilon_{1}}\right\|_{L^{2}(0,2 \pi)},
\end{aligned}
$$

where $\delta_{2}=\frac{1}{4}\left(\frac{p-\epsilon_{1}}{k^{2}}-1\right)>0$. Hence, as $\|v\| \rightarrow \infty, I(v) \rightarrow-\infty$.

By (1.7) and (1.8), we can show as in the proof of Theorem 1.1 that $I$ satisfies the (PS) condition. Consequently, the functional $I$ satisfies the hypotheses of the saddle point theorem. Hence, using Lemma 1.1 of [22], if $I$ has finite critical points which are all nondegenerate, then there exists a critical $x^{*}$ with Morse index equal to $\operatorname{dim} V=k+1 \geq 2$. Moreover, using that 0 is a local minimum of $I$ and $I(0)=0$, it follows that $x^{*}$ is nontrivial. On the other hand, by Lemma $3.2, I$ has a nontrivial nonnegative solution $x^{+}$and a nontrivial nonpositive solution $x^{-}$. Since $x^{+}$and $x^{-}$are all nondegenerate and of mountain pass type, by [19] it follows that $x^{+}$and $x^{-}$are all of Morse index less that or equal to 1 . Then

$$
x^{*} \neq x^{+}, x^{*} \neq x^{-} .
$$


Now, similar as the arguments in the proof of Theorem 1.2(i), we can take $\tau>0$ small enough and $\rho \in\left(0, \rho_{0}\right), r \in\left(0, r_{1}\right), d \in\left(0, d_{1}\right)$ and $R \geq R_{0}$ such that (3.20) holds and

$$
B_{\tau}\left(x^{*}\right) \cap B_{r}\left(x^{+}\right)=\emptyset, B_{\tau}\left(x^{*}\right) \cap B_{d}\left(x^{-}\right)=\emptyset, B_{\tau}\left(x^{*}\right) \cap B_{\rho}=\emptyset
$$

and

$$
\bar{B}_{\tau}\left(x^{*}\right), \bar{B}_{\rho}, \bar{B}_{r}\left(x^{+}\right), \bar{B}_{d}\left(x^{-}\right) \subset B_{R} .
$$

By the additivity and excision properties of the Leray-Schauder degree, we have

$$
\begin{aligned}
\operatorname{deg}\left(\nabla I, B_{R}, 0\right)= & \operatorname{deg}\left(\nabla I, B_{\tau}\left(x^{*}\right), 0\right)+\operatorname{deg}\left(\nabla I, B_{\rho}, 0\right)+\operatorname{deg}\left(\nabla I, B_{r}\left(x^{+}\right), 0\right) \\
& +\operatorname{deg}\left(\nabla I, B_{d}\left(x^{-}\right), 0\right) \\
& +\operatorname{deg}\left(\nabla I, B_{R} \backslash\left(\overline{B_{\tau}\left(x^{*}\right) \cup B_{\rho} \cup B_{r}\left(x^{+}\right) \cup B_{d}\left(x^{-}\right)}\right), 0\right) .
\end{aligned}
$$

Note that all the critical points of $I$ are nongenerate, we can see that

$$
\left|\operatorname{deg}\left(\nabla I, B_{\tau}\left(x^{*}\right), 0\right)\right|=1 .
$$

Then, using Lemmas $3.1,3.2,3.4$ and the excision property of the LeraySchauder degree, we obtain

$$
\operatorname{deg}\left(\nabla I, B_{R} \backslash\left(\overline{B_{\rho} \cup B_{r}\left(x^{+}\right) \cup B_{d}\left(x^{-}\right)}\right), 0\right) \neq 0 .
$$

So, by the existence property of the Leray-Schauder degree it follows that there exists $x_{4} \in B_{R} \backslash\left(\overline{B_{\tau}\left(x^{*}\right) \cup B_{\rho} \cup B_{r}\left(x^{+}\right) \cup B_{d}\left(x^{-}\right)}\right)$such that $\nabla I\left(x_{4}\right)=0$. Thus problem (1.1) has at least four nontrivial solutions: $x^{+}, x^{-}, x^{*}, x_{4}$.

Before proving Theorem 1.2(iii), we recall a global version of the LyapunovSchmidt method.

Lemma 3.6 ([3]). Let $H$ be a real separable Hilbert space. Let $V$ and $W$ be closed subspaces of $H$ such that $H=V \oplus W$. Assume that $J \in C^{1}(H, \mathbb{R})$. If there are $\mu>0$ and $\tau>1$ such that

$\left[\nabla J\left(v+w_{1}\right)-\nabla J\left(v+w_{2}\right)\right]\left(w_{1}-w_{2}\right) \geq \mu\left\|w_{1}-w_{2}\right\|^{\tau}$ for all $v \in V, w_{1}, w_{2} \in W$,

then we have the following.

(i) there exists $\psi \in C(V, W)$ such that

$$
J(v+\psi(v))=\min _{w \in W} J(v+w) .
$$

Moreover, $\psi(x)$ is the unique member of $W$ such that

$$
[\nabla J(v+\psi(v)](w)=0 \text { for all } w \in W
$$

(ii) if we define $\bar{J}(v)=J(v+\psi(v))$, then $\bar{J} \in C^{1}(V, \mathbb{R})$ and

$$
[\nabla \bar{J}(v)]\left(v_{1}\right)=\left[\nabla J(v+\psi(v)]\left(v_{1}\right) \text { for all } v, v_{1} \in V\right.
$$

(iii) An element $v \in V$ is a critical point of $\bar{J}$ if and only if $v+\psi(v)$ is a critical point of $J$; 
(iv) Let $\operatorname{dim} X<\infty$ and $P$ be the projection onto $V$ across $W$. Let $S \subset V$ and $D \subset H$ be open bounded regions such that

$$
\{v+\psi(v) \mid v \in S\}=D \cap\{v+\psi(v) \mid v \in V\} .
$$

If $\nabla \bar{J}(v) \neq 0$ for $v \in \partial S$, then

$$
\operatorname{deg}(\nabla \bar{J}, S, 0)=\operatorname{deg}(\nabla J, D, 0) ;
$$

(v) If $x_{0}=v_{0}+w_{0}$ is a critical point of mountain pass type of $J$, then $v_{0}$ is a critical point of mountain pass type of $\bar{J}$.

Proof of Theorem 1.2(iii). Define the functional $\eta(w): W \rightarrow \mathbb{R}$ by

$$
\eta(w)=\int_{0}^{1}\left[|\dot{w}|^{2}-\beta w^{2}\right] d t, \forall w \in W .
$$

Clearly, by $\int_{0}^{2 \pi}|\dot{w}(t)|^{2} d t \geq(k+1)^{2}\|w\|_{2}^{2}$ for all $w \in W$, we have

$$
\eta(w) \geq \int_{0}^{1}\left[(k+1)^{2}-\beta\right] w^{2} d t \geq 0, \forall w \in W .
$$

We claim that there exist $\mu, M>0$ such that

$$
\eta(w) \geq \mu\|w\|^{2},\|w\| \geq M
$$

Indeed, we assume, by contradiction, that (3.28) doesn't hold. Then there exists a sequence $\left\{w_{n}\right\} \subset W$ with $\left\|w_{n}\right\| \rightarrow \infty$ such that $\frac{\eta\left(w_{n}\right)}{\left\|w_{n}\right\|^{2}} \rightarrow 0$ as $n \rightarrow \infty$. Let $y_{n}=\frac{w_{n}}{\left\|w_{n}\right\|}$. Then $\left\|y_{n}\right\|=1$. Passing, if necessary, to a subsequence we assume that $y_{n} \rightarrow y_{0} \in W$ weakly and $y_{n} \rightarrow y_{0}$ in $C[0,2 \pi]$. By $y_{n}, y_{0} \in W$ it follows that

$$
0 \leq \eta\left(y_{0}\right) \leq \liminf \eta\left(y_{n}\right)=0
$$

which implies that

$$
0=\eta\left(y_{0}\right) \geq \int_{0}^{2 \pi}\left[\dot{y}_{0}^{2}-(k+1)^{2} y_{0}^{2}\right] d t \geq 0 .
$$

Thus $y_{0}$ is an eigenfunction corresponding to $(k+1)^{2}$. Note that $(1.9),(3.27)$ and (3.29) imply that $y_{0} \equiv 0$. A contradiction. Thus (3.28) holds. Now by the mean value theorem and (1.9) it follows that

$$
\begin{aligned}
{\left[\nabla I(v+w)-\nabla I\left(v+w_{1}\right)\right]\left(w-w_{1}\right) } & \geq \int_{0}^{2 \pi}\left[\left|\dot{w}-\dot{w}_{1}\right|^{2}-\beta\left(w-w_{1}\right)^{2}\right] d t \\
& \geq \mu\left\|w-w_{1}\right\|^{2} .
\end{aligned}
$$

Then by Lemma 3.6 there exists $\psi: V \rightarrow W$ such that

$$
I(v+\psi(v))=\min _{w \in W} I(v+w) .
$$

Moreover, $\psi(v)$ is the unique element of $W$ such that

$$
[\nabla I(v+\psi(v))](w)=0 \text { for all } w \in W .
$$


Define $\bar{I}: V \rightarrow \mathbb{R}$ by

Then $\bar{I}$ is of class $C^{1}$, and

$$
\bar{I}(v)=I(v+\psi(v))
$$

$$
[\nabla \bar{I}(v)]\left(v_{1}\right)=[\nabla I(v+\psi(v))(x)]\left(x_{1}\right) \text { for all } v, v_{1} \in V \text {. }
$$

By (3.25), I(v) $\rightarrow-\infty$ as $\|v\| \rightarrow \infty$. Then in view of $\bar{I}(v) \leq I(v)$, we can obtain that

$$
\bar{I}(v) \rightarrow-\infty \text { as }\|v\| \rightarrow \infty .
$$

Since $V$ is of finite dimension, there exists $v_{0} \in V$ such that

$$
\bar{I}\left(v_{0}\right)=\max _{v \in V} I(v+\psi(v)) .
$$

Then $x_{0}=v_{0}+\psi\left(v_{0}\right)$ is a critical point of $I$, i.e., $\nabla I\left(x_{0}\right)=0$. Suppose that $v_{0}$ is an isolated critical point of $\bar{I}$, so $x_{0}$ is an isolated critical point of $I$. By (3.30), $v_{0}$ is a strictly local maximum of the functional $\bar{I}$. Then there exists $\hat{v}$ in some neighborhood $S_{0}$ of $v_{0}$ such that $\bar{I}(\hat{v})<\bar{I}\left(v_{0}\right)$, i.e.,

$$
I(\hat{v}+\psi(\hat{v}))<I\left(v_{0}+\psi\left(v_{0}\right)\right),
$$

which means that $x_{0}$ can't be local minimum of the functional $I$. Thus $x_{0}$ is nontrivial. On the other hand, if we denote $x^{+}=v^{+}+\psi\left(v^{+}\right)$, then by Lemma 3.2 we can see that $v^{+}$is a critical point of mountain pass type of $\bar{I}$, which implies $x_{0} \neq x^{+}$. Similarly, $x_{0} \neq x^{-}$. Furthermore, denoting $B_{\sigma}\left(x_{0}\right)=\{x \in$ $\left.H_{[0,2 \pi]}^{1} \mid\left\|x-x_{0}\right\| \leq \sigma\right\}$, by (3.30) there exists $\sigma_{0}>0$ small such that

$$
\operatorname{deg}\left(\nabla \bar{I},\left.B_{\sigma}\left(x_{0}\right)\right|_{V}, 0\right)=(-1)^{k} \text { for all } 0<\sigma \leq \sigma_{0} .
$$

Hence by Lemma 3.6 it follows that

$$
\operatorname{deg}\left(\nabla I, B_{\sigma}\left(x_{0}\right), 0\right)=(-1)^{k} \text { for all } 0<\sigma \leq \sigma_{0} .
$$

Now similar arguments as in the proof of Theorem 1.2(ii) implies that there exists at least a nontrivial critical point $x_{4}$ of $I$ that different from $x_{0}, x^{+}, x^{-}$. This completes the proof.

Acknowledgments. The authors express their sincere thanks to Prof. Yong Li for his instructions and many invaluable suggestions. This work is partially supported by NSFC Grant(11101178, 11471067), NSFJP Grant(20130522095JH, 201215184), FSIIP of Jilin University(201103203) and the 985 Program of Jilin University.

\section{References}

[1] H. Amann, A note on degree theory for gradient mappings, Proc. Amer. Math. Soc. 85 (1982), no. 4, 591-595.

[2] G. Barletta and N. S. Papageorgiou, Periodic problems with double resonance, NoDEA Nonlinear Differential Equations Appl. 19 (2012), no. 3, 303-328.

[3] A. Castro and J. Cossio, Multiple solutions for a nonlinear Dirichlet problem, SIAM J. Math. Anal. 25 (1994), no. 6, 1554-1561.

[4] X. J. Chang and Q. D. Huang, Two-point boundary value problems for Duffing equations across resonance, J. Optim. Theory Appl. 140 (2009), no. 3, 419-430. 
[5] X. J. Chang and Y. Li, Existence and multiplicity of nontrivial solutions for semilinear elliptic Dirichlet problems across resonance, Topol. Methods Nonlinear Anal. 36 (2010), no. 2, 285-310.

[6] X. J. Chang, Y. Li, and S. G. Ji, Nonresonance conditions on the potential with respect to the Fučík spectrum for semilinear Dirichlet problems, Z. Angew. Math. Phys. 61 (2010), no. 5, 823-833.

[7] X. J. Chang and Y. Qiao, Existence of periodic solutions for a class of p-Laplacian equations, Bound. Value Probl. 2013 (2013), 11 pp.

[8] D. G. Costa and A. S. Oliveira, Existence of solution for a class of semilinear elliptic problems at double resonance, Bol. Soc. Brasil. Mat. 19 (1988), no. 1, 21-37.

[9] D. Del Santo and P. Omari, Nonresonance conditions on the potential for a semilinear elliptic problem, J. Differential Equations 108 (1994), no. 1, 120-138.

[10] T. Ding, R. Iannacci, and F. Zanolin, Existence and multiplicity results for periodic solutions of semilinear Duffing equations, J. Differential Equations 105 (1993), no. 2, 364-409.

[11] C. L. Dolph, Nonlinear integral equations of the Hammerstein type, Trans. Amer. Math. Soc. 66 (1949), 289-307.

[12] P. Drábek, Landesman-Lazer conditions for nonlinear problems with jumping nonlinearities, J. Differential Equations 85 (1990), no. 1, 186-199.

[13] P. Habets and G. Metzen, Existence of periodic solutions of Duffing equations, J. Differential Equations 78 (1989), no. 1, 1-32.

[14] P. Habets, P. Omari, and F. Zanolin, Nonresonance conditions on the potential with respect to the Fuik spectrum for the periodic boundary value problem, Rocky Mountain J. Math. 25 (1995), no. 4, 1305-1340.

[15] C. Fabry and A. Fonda, Periodic solutions of nonlinear differential equations with double resonance, Ann. Mat. Pura Appl. (4) 157 (1990), 99-116.

[16] A. Fonda, On the existence of periodic solutions for scalar second order differential equations when only the asymptotic behaviour of the potential is known, Proc. Amer. Math. Soc. 119 (1993), no. 2, 439-445.

[17] A. Fonda and P. Habets, Periodic solutions of asymptotically positively homogeneous differential equations, J. Differential Equations 81 (1989), no. 1, 68-97.

[18] D. Y. Hao and S. W. Ma, Semilinear Duffing equations crossing resonance points, J. Differential Equations 133 (1997), no. 1, 98-116.

[19] H. Hofer, The topological degree at a critical point of mountain pass type, Nonlinear functional analysis and its applications, Part 1 (Berkeley, Calif., 1983), 501-509, Proc. Sympos. Pure Math., 45, Part 1, Amer. Math. Soc., Providence, RI, 1986.

[20] R. Iannacci and M. N. Nkashama, Unbounded perturbations of forced second order ordinary differential equations at resonance, J. Differential Equations 69 (1987), no. 3, $289-301$.

[21] E. M. Landesman and A. C. Lazer, Nonlinear perturbations of linear elliptic boundary value problems at resonance, J. Math. Mech. 19 (1969/1970), 609-623.

[22] A. C. Lazer and S. Solimini, Nontrivial solutions of operator equations and morse indices of critical points of min-max type, Nonlinear Anal. 12 (1988), no. 8, 761-775.

[23] W. B. Liu and Y. Li, Existence of $2 \pi$-periodic solutions for the non-dissipative Duffing equation under asymptotic behaviors of potential function, Z. Angew. Math. Phys. 57 (2006), no. 1, 1-11.

[24] J. Mawhin and M. Willem, Critical Point Theory and Hamiltonian Systems, SpringVerlag, Berlin, 1989.

[25] L. Nirenberg, Topics in Nonlinear Functional Analysis, Courant Institute of Mathematical Sciences, New York University, New York, 1974.

[26] P. Omari and F. Zanolin, Nonresonance conditions on the potential for a second-order periodic boundary value problem, Proc. Amer. Math. Soc. 117 (1993), no. 1, 125-135. 
[27] N. S. Papageorgiou and V. Staicu, Multiple nontrivial solutions for doubly resonant periodic problems, Canad. Math. Bull. 53 (2010), no. 2, 347-359.

[28] J. Su and L. Zhao, Multiple periodic solutions of ordinary differential equations with double resonance, Nonlinear Anal. 70 (2009), no. 4, 1520-1527.

[29] M. R. Zhang, Nonresonance conditions for asymptotically positively homogeneous differential systems: the Fučík spectrum and its generalization, J. Differential Equations 145 (1998), no. 2, 332-366.

[30] X. Zhao and X. J. Chang, Existence of anti-periodic solutions for second-order ordinary differential equations involving the Fuč ik spectrum, Bound. Value Probl. 2012 (2012), $12 \mathrm{pp}$.

Hua CaI

College of Mathematics

JILIN UNIVERSITY

Changchun 130012, P. R. China

E-mail address: caihua@mail.jlu.edu.cn

Xiaojun Chang

School of Mathematics and Statistics

Northeast Normal University

Changchun 130024, P. R. China

AND

College of Mathematics

JILIN UNIVERSITY

Changchun 130012, P. R. China

E-mail address: changxj1982@hotmail.com

XIN ZhaO

College of Information Technology

JiLin Agricultural University

Changchun 130118, P. R. China

E-mail address: jlndzx@sina.com 